3. Which of the following best describes the optimal site on the stomach wall that is sutured to the abdominal wall during laparoscopic-assisted gastropexy?

(A) A point directly over the pylorus on the ventral side of the stomach
(B) A point $10 \mathrm{~cm}$ from the pylorus on the greater curvature of the stomach

(C) A point $5 \mathrm{~cm}$ from the pylorus midway between the greater and lesser curvature of the stomach

(D) A point $6 \mathrm{~cm}$ from the pylorus on the lesser curvature of the stomach

\section{Getting the most out of your laparoscopic kit: feline}

\section{Philip Lhermette}

Most practices invest in laparoscopic equipment in order to carry out bitch spays. However the same benefits apply to cats and laparoscopic cat spays can be performed routinely in general practice, not only benefitting our patients but also increasing practice profitability. Indeed cats make ideal laparoscopic patients having a relatively expandable abdomen and less falciform fat.

Most of the procedures that are carried out laparoscopically in dogs can also be done in cats and clients are very open to having minimally invasive procedures rather than open laparotomy, especially for diagnostic procedures such as organ biopsy. The same basic equipment can be used as in canine laparoscopy, although a smaller, 2.7- $\mathrm{mm}$ endoscope is less unwieldy than the more common 5-mm endoscope, and if a lot of feline procedures are undertaken, smaller $3-\mathrm{mm}$ instrumentation can be an advantage. With the addition of very little additional instrumentation a wide range of other procedures can be performed including rhinoscopy, urethrocystoscopy, fistuloscopy, video-otoscopy, and thoracoscopy - further improving the profitability of the equipment.

So what other procedures can be done in the abdomen minimally invasively?

Exploratory laparoscopy
- Liver biopsy
Kidney biopsy
- - Lancreatic biopsy
- Splenic biopsy
- Full-thickness intestinal biopsy
- Ovariectomy/ovariohysterectomy
- Lryptorchidectomy
- Intestinal/gastric foreign body removal
- Examination of the gall bladder and biliary tree
- Intra-abdominal neoplasia staging/resection
- Investigation of intra-abdominal trauma

- Nephrectomy

- Other operative surgery.

\section{ORGAN BIOPSIES}

Commonly performed biopsy procedures include liver, pancreatic and full-thickness bowel biopsies. Cholecystocentesis and kidney biopsies can also be performed under laparoscopic guidance and haemostasis can be achieved effectively under direct visualization.

\section{CRYPTORCHIDECTOMY}

Cryptorchidectomy can also be carried out in the cat in a similar fashion to that described for the dog.

\section{LAPAROSCOPIC-ASSISTED CYSTOSCOPY}

Laparoscopic-assisted cystoscopy is the procedure of choice for male dogs and cats or where large stones are to be removed. Bladder polyps or transitional cell carcinomas may be ablated using a diode laser via this approach. Visualization is greatly improved over traditional open cystotomy and postoperative pain is reduced.

The abdomen is insufflated and a primary camera port is placed at or around the umbilicus. A second port is placed at the midline directly over the apex of the bladder and the bladder wall is grasped and bought up to the abdominal incision where it is sutured to the abdominal wall to form a seal. The endoscope is transferred into an incision made in the bladder wall to inspect the interior of the bladder and instruments can be passed alongside to retrieve stones etc.

At the end of the procedure, the bladder wall is sutured in the normal fashion, the stay sutures are removed and the bladder is returned to the abdomen. The abdomen is fully deflated and all ports and catheters are removed. Closure is routine.

\section{KEY LEARNING OBJECTIVES}

- Be able to list, describe and compare the advantages and disadvantages of minimally invasive laparoscopic procedures in the cat

- Identify the main laparoscopic procedures that are carried out routinely in the cat using basic laparoscopic equipment

- Describe and discuss the procedure for laparoscopic-assisted cystoscopy in the cat 


\section{MULTIPLE CHOICE QUESTIONS}

1. Which of the following is NOT a feature of the cat as a candidate for laparoscopy, compared to the dog?
(A) More operative space than an equivalent weight dog
(B) Less falciform fat
(C) Increased risk of splenic injury
(D) Increased risk of cannula slippage

2. Which of the following best describes the procedure for laparoscopic-assisted full-thickness bowel biopsy?
(A) A loop of bowel is grasped within the abdomen and a biopsy performed with monopolar biopsy forceps
(B) A loop of bowel is bought up to the abdominal wall and a biopsy performed with a punch biopsy
(C) A small loop of bowel is grasped, brought through the abdominal wall and a biopsy performed by incision or punch biopsy

(D) A large loop of bowel is brought through the abdominal wall and several biopsy specimens taken at different sites

3. Which of the following best describes the procedure for laparoscopic-assisted cystoscopy in the cat?
(A) The abdomen is insufflated and an endoscope is passed through a cannula in the abdominal wall and into the bladder
(B) The bladder is held against the abdominal wall with forceps and a cannula is passed directly through into the lumen of the bladder
(C) The bladder is distended with saline and a cannula is inserted through the abdominal wall directly into the bladder
(D) The bladder wall is grasped at the apex and bought up to the linea alba where it is sutured to the incision

\section{Angular limb deformities}

\section{Kevin Parsons}

Angular limb deformities most commonly affect the radius and ulna. Underlying causes include:

- Premature closure of the proximal or distal physis of the ulna

- Asymmetric closure of the proximal or distal physis of the radius

- A combination of both.

Aims of treatment include alleviating pain (most commonly achieved by addressing joint incongruity), correcting bony deformities that impair function and addressing clinically significant limb shortening. Growth potential also needs to be factored into planning.

Thorough investigations, including the clinical history, orthopaedic examination and diagnostic imaging, are necessary to evaluate the case fully. Traditionally the classification of the type and extent of the deformity and subsequent surgical planning have been performed from radiographic studies. More recently computed tomography (CT) has been used to enable quantification of torsion on preoperative imaging, permitting more objective presurgical planning.

A number of factors need to be considered when formulating a treatment plan including:

\footnotetext{
Whether surgery is necessary at all

The timing of surgery

The surgical methods employed

- Which components of the deformity to address (joint incongruity and/or bone deformity).
}

When planning corrective surgery, accurate localization and quantification of the deformity is critical. It is well described that the determination of the centre of rotation of angulation (CORA) is important to identify where to centre osteotomies. If two CORAs exist, two osteotomies may be required. In addition to determining frontal and sagittal plane components of deformities, torsion must also be considered.

Once the decision has been made that surgery is indicated to straighten the affected bone, one needs to decide whether to perform acute or a gradual correction (most commonly using a circular external fixator). The creation of three-dimensional (3D) anatomical models from CT data allows a rehearsal surgery which contributes to successful planning and execution of surgery.

Techniques reported for addressing angular limb deformities include acute correction via osteotomy stabilized with either external or internal fixation. Alternatively, in the immature patient, gradual correction using llizarov methodology can be considered. When performing acute corrections with internal fixation the ability to make postoperative adjustments is limited.

The effect of addressing bony deformities on the surrounding soft tissues needs to be considered. In some cases of marked pro-curvatum, the caudal soft tissues may not tolerate acute lengthening.

Recently the use virtual 3D surgical planning has become accessible. Working with CAD software, imaging data is processed to create on screen 3D models. This then allows 'virtual' surgery to be performed, with the creation of osteotomies and realignment of bone segments. Once completed on the virtual screen, cutting and repositioning guides can be created. These guides allow precise correction of the deformity during surgery with predictable bone realignment to match that of the virtual surgery performed earlier. 